\title{
Pigeon reaction time, Hick's law, and intelligence
}

\author{
CORYN VICKREY and ALLEN NEURINGER \\ Reed College, Portland, Oregon
}

\begin{abstract}
Pigeons' choice reaction times (RTs) increased as a linear function of $\log _{2}$ number of potential target stimuli (Experiments 1-3), as would be predicted by Hick's law. The values of intercepts and slopes decreased with training (Experiments 2 and 3) and with differential reinforcement of short RTs under percentile reinforcement contingencies (Experiment 3). RT functions obtained from human subjects were also consistent with Hick's law, but slopes for pigeons were significantly lower than those for humans (Experiments 4 and 5). These results extend the generality of Hick's law to pigeons but are inconsistent with Jensen's claim that the parameters of the Hick function are related to intelligence.
\end{abstract}

Reaction time (RT) is often used to measure cognitive processes in human subjects (Luce, 1986; Posner, 1986). One common procedure is for subjects to sit in front of a display that contains one, two, four, or eight potentially lighted bulbs, or potential targets (PTs), each with a small button beneath it. Also present is a centrally located button. The subject must press the center button to initiate a trial. After a variable interval, a randomly selected bulb is illuminated, and the subject must press the associated button as quickly as possible. In the one-bulb case, only a single PT can be lighted, all others being masked by a metal plate; in the two-bulb case, two PTs are available, with the other six masked, and one of the two, randomly selected, is illuminated; and so on (Jensen, 1980, 1982). The obtained relationship, often referred to as Hick's law, is that RT varies as a linear function of $\log _{2} \mathrm{PT}$, or bits of information, with $1 \mathrm{PT}=0$ bits, $2 \mathrm{PTs}=1 \mathrm{bit}, 4 \mathrm{PTs}=2$ bits, and 8 PTs $=3$ bits (Hick, 1952; Hyman, 1953).

Jensen reported negative correlations between IQ and both the intercept and the slope of the Hick function (Jensen, 1980, 1987; Jensen \& Munro, 1979). People scoring high on tests of IQ respond faster (have shorter RTs), on average, than people scoring lower on those tests (difference in intercepts), and RTs of high-IQ subjects increase less rapidly with increasing PTs than do those of the low-IQ individuals - that is, the difference in RT between high- and low-IQ subjects increases with number of PTs, or complexity (dif-

Portions of these data were reported by C.V. in a Reed College undergraduate thesis and were presented to the Society for the Quantitative Analysis of Behavior, May 1996, San Francisco. This research was supported, in part, by grants from the National Science Foundation to A.N. We thank Gene Olson and Chris Deiss for animal care and John Hopson and Chris Deiss for programming assistance. Correspondence concerning this article should be addressed to A. Neuringer, Psychology Department, Reed College, Portland, OR 97202 (e-mail: allen.neuringer@ reed.edu). ference in slopes). RT increases with bits of information, according to this interpretation, because each binary decision takes a constant amount of time and choosing among eight PTs takes three binary decisions, whereas choosing between two PTs takes only one (Jensen, 1980). Intelligence is correlated with the Hick function, because high intelligence depends on rapid processing of information (Jensen, 1980). Considerable empirical evidence has been offered in support of these claims (e.g., Carroll, 1987; Jensen, 1987; Neubauer, 1990), although contradictory findings have also been reported (e.g., Beauducel \& Brocke, 1993).

The simplicity of Hick's procedure has enabled comparisons across diverse human populations and suggests that the procedure could be used to compare RTs and, possibly, intelligence across species, although there have been few such studies. In one notable case, Laursen (1977) showed that, in vervet monkeys, the form of the Hick function is similar to that in humans.

The goals of the present research are three. The first is to test whether Hick's law applies to pigeons. The second is to determine whether the slope and intercept of the Hick function change with experience. This question is important, because Jensen (1980) reports that, in his studies, practice and experience leave these parameters unaffected-a result consistent with the claim that intelligence involves $g$, an inherited general factor-but there is conflicting evidence (Teichner \& Krebs, 1974; Widaman \& Carlson, 1989). Animal models may be especially useful in testing claims about experience, because of their controlled rearing and experimental histories. The third goal is to determine whether slopes from pigeon subjects are steeper than those from human subjects. For cross-species comparisons, slope will be more important than intercept (or average RT), because the latter is affected by particulars of apparatus and procedure, as well as by the subject's anatomy. If $g$ has cross-species relevance, steeper slopes would be expected from pigeons than from people. As will be dis- 
cussed later, alternatives to the general factor theory of intelligence lead to different predictions.

\section{EXPERIMENT 1 A Test of Hick's Law in Pigeons}

\begin{abstract}
Method
Subjects. Five experimentally naive adult Birmingham Roller pigeons were maintained at $85 \%$ of their free-feeding body weights. Apparatus. Each of five Gerbrands pigeon chambers $(27.9 \times$ $27.9 \times 29.2 \mathrm{~cm})$ consisted of a front wall with three operant response keys (not used in the present experiment) and a food hopper that provided access to mixed grain. An overhead light provided illumination, and a small speaker provided auditory feedback. At a right angle to the front wall was a 33-cm Apple color monitor with a touch screen, consisting of a Carroll Touch $30.5-\mathrm{cm}$ Smartframe touch-sensitive bezel that enabled determination of the location and timing of pecks to the monitor. Mounted $0.6 \mathrm{~cm}$ in front of the screen was a plastic sheet, to protect the birds' beaks from the monitor's hard surface. A movable wooden perch parallel to the monitor was positioned according to the size of the bird, so as to provide easy access to the screen. The chambers were individually enclosed in wooden boxes and were connected to five Macintosh LC475 computers.
\end{abstract}

Procedure. Preliminary training consisted of autoshaping the pigeons to peck a black square $(1.3 \mathrm{~cm}$ per side), then to peck at a green star $(2.5 \mathrm{~cm}$ diameter $)$, and finally to peck the black square and then the green star to gain access to grain in the food hopper. In the experimental procedure that followed, a filled black $1.3-\mathrm{cm}$ square was presented in the center of the screen; it will be referred to as the center stimulus. Equally spaced in a virtual circle (diameter of $7.6 \mathrm{~cm}$ ) surrounding the center stimulus were one, two, four, or eight open squares of equal size, the PTs. In the one-PT condition, a single open box was presented simultaneously with the center stimulus; in the two-PT condition, there were two open boxes; and so on. One peck to the center stimulus caused one of the PTs to be darkened (filled black), so that it looked identical to the center stimulus; this darkened alternative now becoming the target. One peck to the target resulted in the appearance of a reinforcing star (the green star) in the center of the screen, and a peck to the star produced food. In brief, pecking the center stimulus darkened the target, pecking the target produced the green star, and pecking the star resulted in food. Some further details follow. After the center stimulus was pecked to start a trial, that stimulus disappeared, the RT timer was started, and a $1000-\mathrm{Hz}$ tone sounded for $0.1 \mathrm{sec}$. A correct response was a peck at the darkened target within the boundary of the accept area, an area that extended $1.27 \mathrm{~cm}$ past the edges of each stimulus. (As will be discussed below, a programming error caused the right boundary of all the stimuli to be offset, thereby requiring correct responses to be to the right half of the stimulus or just outside the right boundary.) Correct responses to the target stopped the $\mathrm{RT}$ timer and were accompanied by a $1800-\mathrm{Hz}$ tone for $0.1 \mathrm{sec}$. Responses to the reinforcing star produced a $400-\mathrm{Hz}$ tone for $0.2 \mathrm{sec}$ and access to food in the hopper for a designated duration that was individualized for each bird, to maintain appropriate weights (the duration usually being between 1.5 and $2.5 \mathrm{sec}$ ). During food reinforcement, the hopper and houselight were on.

A response outside the accept area of the target caused a different tone sequence (a $600-\mathrm{Hz}$ tone for $0.1 \mathrm{sec}$, a $500-\mathrm{Hz}$ tone for $0.1 \mathrm{sec}$, and $\mathrm{a} 400-\mathrm{Hz}$ tone for $0.2 \mathrm{sec}$ ); the green star did not appear, and the same trial (same number of PTs in exactly the same locations and same location of the target) was repeated as a correction trial. Such incorrect responses and those made during correction trials were excluded from calculations of RT. Locations of PTs (open boxes) and targets (black box) were randomized among eight possible locations around the virtual circle. The one-, two-, four-, and eight-PT conditions were quasi-randomized (eight blocks of eight trials per session with two trials of each PT condition in each block).
During the first session following preliminary training, the pigeons were presented with only the one-PT condition. During the next session, one- and two-PT conditions were intermixed, and so on until the pigeons were reliably responding with all eight alternatives present. These sessions terminated after 64 correct trials.

The experiment started in the next session with the one-, two-, four-, and eight-PT conditions intermixed randomly, as was described above. Eleven sessions were provided, each session containing 64 correct responses, 16 per PT condition. After the 11 th session, conditioned reinforcers were introduced so that the number of trials per session could be increased to 128 and an additional 14 sessions provided. Conditioned reinforcers were the same as the food reinforcers, except that the hopper was activated for only $0.1 \mathrm{sec}$, this duration being too short to permit the pigeons to eat any food. Food and conditioned reinforcers were equally probable $(p=.5)$. One session was provided per day, 5 days per week.

\section{Results and Discussion}

Median RTs were calculated for each subject, based on the initial 200 correct responses in each of the four PT conditions at the beginning of training and, separately, for the last 200 correct trials per PT at the end of training. Arithmetic averages of the 5 birds' median RTs are shown in Figure 1, left, for both initial and last trials (filled crosses and open circles). Least squares best-fitting lines offer good approximations of average performances, with $80 \%$ and $95 \%$ of the variances accounted for (correlation coefficients, $r$, are shown in the figures, and explained variance equals the square of the correlation, or $r^{2}$ ). Slopes of the initial and terminal functions were .015 and $.017 \mathrm{sec} / \mathrm{bit}$, respectively, and intercepts were .412 and $.385 \mathrm{sec}$. The $r$ values for the individual subjects over the last 200 trials were $.89, .93, .94, .94$, and .95 . The slopes and intercepts did not differ significantly between initial and terminal blocks.

RT showed a positive linear relationship to $\log _{2}$ PT, consistent with the findings from people. However, the pigeon slopes were considerably lower than those generally reported for human subjects (Jensen, 1980, 1982). If slope is, as hypothesized, inversely related to intelligence, this finding is puzzling.

\section{EXPERIMENT 2 Blocks of Contiguous Potential Targets}

Three aspects of the procedure in Experiment 1 may have contributed to the pigeons' low slopes. First, in Experiment 1 , locations of PTs and targets were randomized, whereas Jensen and others did not randomize. Second, in human experiments, the locations of the PTs were always contiguous and fixed-in the four-PT condition, for example, the locations, assuming that positions are indicated clockwise from 1 to 8 , were $3,4,5$, and 6-whereas randomization in Experiment 1 resulted in noncontiguous PTs. Third in human experiments, the PT conditions were presented in ascending blocks-for example, 15 trials of the one-PT condition were presented first, then 15 trials of the two-PT condition, and so on (Jensen, 1982). In Experiment 1, there was no blocking, and the PT conditions were intermixed in quasi-random order (e.g., one PT, eight PTs, two PTs, eight PTs, four PTs, etc.) 


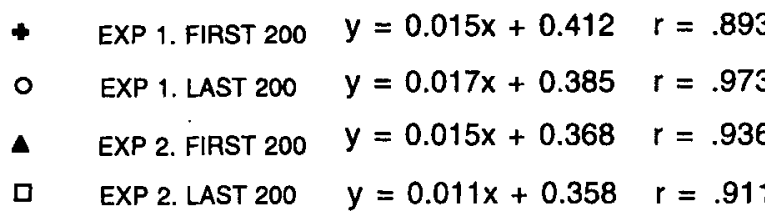

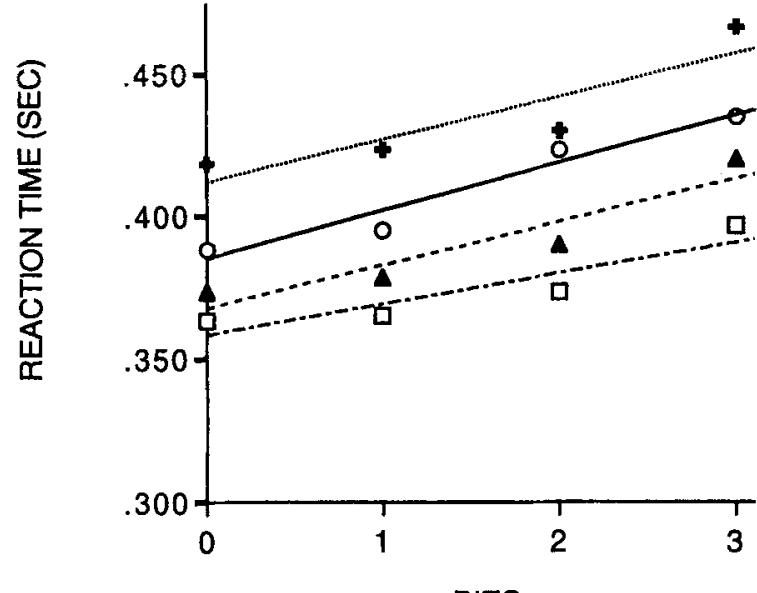

BITS
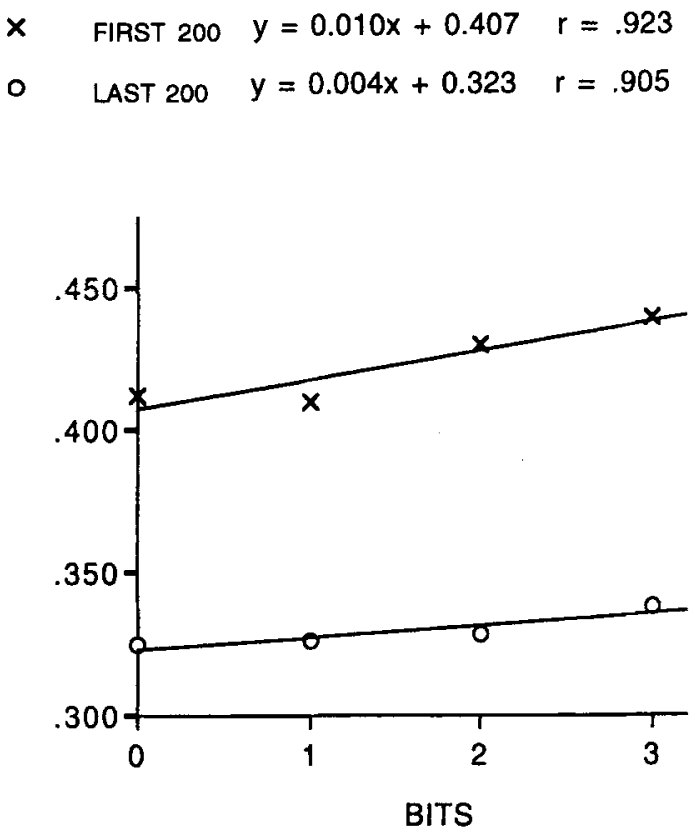

Figure 1. The left graph shows average reaction times for the 5 subjects in Experiments 1 and 2 as a function of $\log _{2}$ of the number of potential targets (PTs) or bits of information. The first 200 trials per PT are indicated by filled crosses and triangles, and the last 200 trials by open circles and squares. The right graph shows average reaction times for the 7 subjects under percentile reinforcement contingencies in Experiment 3, again for the first (Xs) and last (circles) 200 trials per PT.

There were reasons to randomize (see Detterman, 1987 Widaman \& Carlson, 1989). As was indicated above, the Hick function has been interpreted as resulting from a series of binary decisions, but an alternative interpretation is based on results that show that repeating the location of a target stimulus may itself shorten RT (Kornblum, 1969; Luce, 1986). In Jensen's research, repetitions were more likely in the two-PT case than in the four-PT case, and more likely in the four-PT case than in the eight-PT case. The reason is that, if the same two lights are always the only possibilities in the two-PT case, the probability of a light repeating is .5 ; if the same four lights are the only possibilities in the four-PT condition, the repetition probability is .25; and so on. The linear function relating RT to $\log _{2}$ PT may, therefore, have been influenced by different probabilities of repetitions in the different PT conditions. Randomization of stimulus locations in Experiment 1 controlled for repetitions, because probabilities of repetition were equal across the PT conditions.

However, the blocking in the human experiments may have led to the formation of expectancies by the subjects, which would also influence the slopes. To increase the similarity between pigeon and human procedures while avoiding the confounding effects of repetition, we modified the procedure in the following way. Both blocking of PT conditions and contiguity of PT locations were employed (as in the human procedures)-for example, 10 correct responses were required in the four-PT condition, followed by 10 correct in the one-PT condition, then 10 in the eightPT condition, and so on. This blocking permitted forma- tion of the same expectancies as those in the human case. However, the order of the blocks was randomized, and the location of the contiguous PTs, within and without a block, was also randomized. Thus, on Trial 1 within a four-PT block, the PTs might be at Locations 3, 4, 5, and 6; on Trial 2, within the same block, the PTs might be at Locations 6, 7, 8, and 1; and so on. This type of randomization avoided the artifact of location repetition. One additional change was made: A misalignment of the stimuli was corrected midway through this experiment. In brief, Experiment 2 more closely approximated human procedures, while avoiding the potential confounds of location repetition.

\section{Method}

Subjects and Apparatus. The subjects and apparatus were the same as those in Experiment 1.

Procedure. The procedure was similar to that in Experiment 1 with the following differences. Trials with the same number of PTs (one, two, four, or eight) were blocked, rather than randomly distributed, 10 correct trials now constituting one block. There were four blocks of each PT condition per session, the order of blocks being quasi-randomized. In any given trial, PTs were always adjacent to one another, although the particular set of locations was randomly chosen, as was the location of the target. Sessions lasted for 160 correct trials. Food followed correct responses with a .4 probability, the other correct responses producing conditioned reinforcement, as was described in Experiment 1.

After 20 sessions, the programming error was identified that had resulted in the accept coordinates around each stimulus being offset $1.9 \mathrm{~cm}$ to the right. (This offset was also present in Experiment 1.) After correction, an additional 11 sessions were provided. 


\section{Results and Discussion}

Figure 1, left, shows the RT functions, averaged across the 5 subjects' initial (filled triangles) and terminal (open squares) 200 trials per PT condition. RT was again linearly related to bits of information. Slopes and intercepts were lower at the end of the present experiment than at the end of Experiment 1, with only the intercept difference reaching significance $[F(1,4)=11.627, p=.02]$. The individual subjects' $r$ values during the terminal 200 trials were $.78, .78$, $.94, .94$, and .98 . Slopes and intercepts did not differ significantly pre- versus postcorrection of the stimulus alignment.

The main conclusion was that blocking and contiguity of PTs did not increase slopes. On the contrary, the obtained slopes were even more unlike those commonly found in human experiments, where low slopes have been suggested as a correlate of high intelligence.

\section{EXPERIMENT 3 \\ Reaction Time and Percentile Reinforcement Contingencies}

Consequences in operant experiments and payoff matrices in signal detection experiments affect response accuracies and RTs (Luce, 1986). However, in research with Hick's paradigm, explicit RT-based feedback is not provided, and instructions are generally of the form respond as fast and accurately as possible. Such instructions might mean different things to different individuals, and nonhuman animals cannot be verbally instructed. It might be especially important for cross-population or cross-species comparisons to use a procedure in which the contingencies explicitly motivated fast-as-possible responding. In Experiment 3, therefore, a percentile reinforcement contingency was added to the Hick procedure. Under percentile contingencies, developed by Platt and Galbicka (e.g., Galbicka, 1994; Platt,1973), each subject's recent RTs were used to determine an RT threshold. If a current RT was longer than the threshold, it was not reinforced. The percentile contingencies continually "pushed" a subject to respond as fast as possible. The first question in the present experiment was whether explicitly reinforcing fast-as-possible responding would influence slopes. A second goal of the present experiment was to study whether slopes and intercepts changed with practice when speed contingencies selectively reinforced fast responding. As was indicated in the introduction, there is disagreement in the human literature concerning the effects of practice.

\footnotetext{
Method

Subjects. Seven young adult White King pigeons were maintained at $85 \%$ of their free-feeding body weight.

Apparatus. The apparatus was the same as that in Experiments 1 and 2 .

Procedure. The procedure was similar to that in Experiment 2, with the same stimuli presented in the same blocks, contiguity of the PTs, and randomization of location. However, under a percentile reinforcement contingency, reinforcement was contingent on rela-
}

tively short RTs: A subject's response was reinforced only if it was among the fastest $70 \%$ of the subject's recent responses. Arrays were maintained of the last 10 correct responses, separately for the one-, two-, four-, and eight-PT conditions. Because RTs may have differed at different stimulus locations, separate arrays were maintained for each of the eight physical locations. Thus, the number of percentile reinforcement arrays was 32: 4 PT conditions $\times 8$ physical locations. Prior to each response, the appropriate array was ordered from shortest RT to longest. The current response was reinforced only if (1) it was correct (i.e., emitted to the target) and (2) it was faster than the third slowest RT (70th percentile) within the relevant array. Responses that met both the location and the speed criteria were reinforced with the green star. Food occurred with .4 probability, and conditioned reinforcement with .6 probability, as in Experiment 2. Responses that did not meet the speed contingency or were to the wrong location generated downward tones and terminated the trials, with only wrong-location trials being excluded from the arrays and repeated, as in Experiments $l$ and 2 . The RT arrays were initially seeded (prior to the first experimental session only) with 1-sec values, considerably longer than most RTs. Only after at least two sessions did the arrays fill with the subject's RTs. Arrays were maintained across sessions. Preliminary training was similar to that in Experiment 1 . Twenty sessions were provided under the percentile reinforcement contingencies.

\section{Results and Discussion}

Figure 1, right, shows arithmetic averages across the 7 pigeon's median 200 initial and 200 terminal trials. As in Experiments 1 and 2, linear functions relating RT to bits of information provide good approximations of the averaged data, accounting for $85 \%$ and $82 \%$ of the variance. Over the 200 terminal trials, individual $r$ values were $.26, .78, .78$, .92 , and .95 , with two of the subjects' values not being defined, because the slopes were zero. The across-session change is shown in Figure 2, with intercept on top and slope on bottom. Arithmetic averages of the 7 subjects' intercepts and slopes are drawn. Analyses of these data show that the decrease in intercept was highly significant $[F(19,114)=$ $14.40, p=.0000]$ and that the decrease in slope approached significance $[F(19,114)=1.528, p=.0887]$. Thus, when rapid responding was explicitly reinforced, the intercept of the Hick function decreased with practice, and there is some indication that the slope also decreased.

To increase the power of the analysis, we combined across all pigeons (Experiments 1-3) and compared the first versus the last 200 trials. Again, the decrease in intercept was highly significant $[F(1,11)=22.803, p=$ $.0003]$, and the decrease in slope was very close to attaining significance $[F(1,11)=4.627, p=.0545]$.

The other main question was whether explicit reinforcement of relatively short RTs under percentile contingencies would affect slopes. The results show that the slopes were lower than those in the previous two experiments. Average slope during the last 200 trials of the present experiment was $.004 \mathrm{sec} / \mathrm{bit}$, whereas at the end of Experiment 2, it was $.011 \mathrm{sec} / \mathrm{bit}$, a significant difference $[F(1,10)=14.074, p=.0038]$. Thus, contingencies that selectively reinforced fast responding generated lower slopes than did contingencies that were more permissive. 

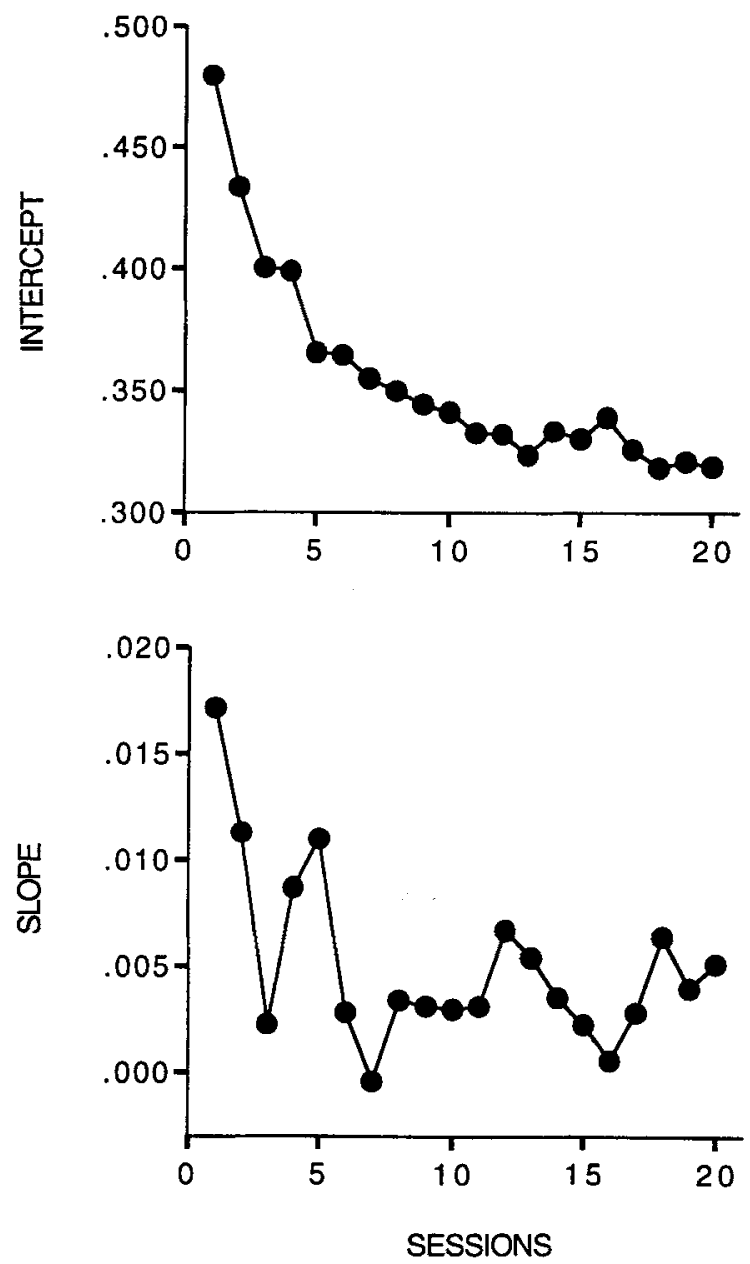

Figure 2. Intercept (top) and slope (bottom) of the Hick function across the 20 sessions of Experiment 3, where percentile reinforcement contingencies were applied. Plotted are the averages for the 7 subjects.

\section{EXPERIMENT 4 Human Touch Reaction Times}

To compare human and pigeon RT functions directly, the procedure in Experiment 2 was repeated with human subjects. That procedure was chosen because of its similarity to those used in previous human studies, while, as was explained above, it avoided the confound of location repetitions.

\section{Method}

Subjects. Six males and 5 females of college age volunteered. Apparatus. A Macintosh II computer and monitor with a touch screen, identical to those used in Experiments 1 and 2, were located on a bench in a laboratory room.

Procedure. The program and contingencies were almost identical to those in Experiment 2. A picture of an eight-PT condition was shown to the subjects, and the task was explained. The subjects were told to touch the black square in the center and then to touch the target when it darkened. As in most other studies with human subjects under the Hick procedure, the subjects were instructed to respond as rapidly as possible while making few errors. There was a short practice session prior to the experimental session, to familiarize the subjects with the touch screen: 10 black squares appeared in random locations, and the subject was instructed to touch them to make them disappear. Immediately following was the experimental procedure from Experiment 2, until 40 correct trials per PT were completed. Reinforcement consisted of points added to a counter in the upper left-hand corner of the screen.

\section{Results and Discussion}

The upward pointing triangles in Figure 3 show median RTs based on the 40 correct trials at each of the four PT conditions. A linear function accounted for $89 \%$ of the variance, with the average slope equaling .034 and the intercept .293 . The individual $r$ values were $.79, .79, .85$, $.85, .87, .90, .91, .94, .97,1.00$, and 1.00 .

Experiments 1-3 showed that intercepts and slopes decreased with experience, and we therefore compared pigeon with human subjects across an equal number of training trials. Since the humans experienced a total of 40 correct trials per PT condition, we used the first 40 correct trials per PT generated by the 5 Birmingham Rollers in Experiment 1 and by the 7 White Kings in Experiment 3. Although the two pigeon functions are difficult to dis-
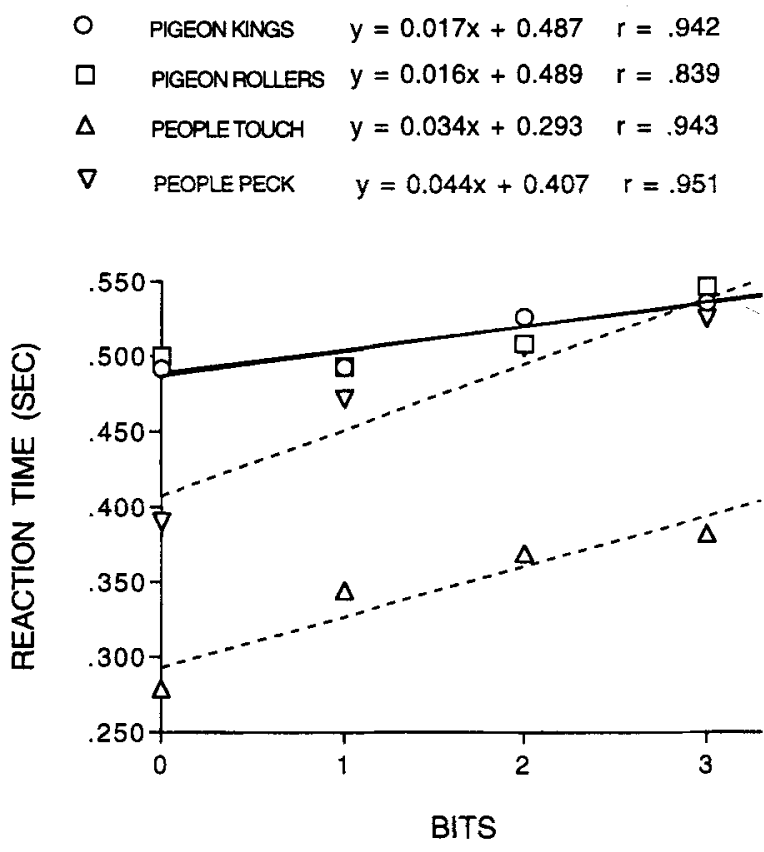

Figure 3. Average reaction times for 5 White King pigeons in Experiment 1, 7 Birmingham Roller pigeons in Experiment 3,11 human subjects in Experiment 4 who touched the monitor, and 10 human subjects in Experiment 5 who "pecked" the monitor. The $x$-axis shows $\log _{2}$ of the number of potential targets (PTs), or bits. In all cases, averages are based on the first 40 correct trials per PT. 
criminate (two lines are drawn), circles represent the averages for the Birmingham Rollers, and squares those for the White Kings. Slopes for the two groups of pigeons over the first 40 responses per PT were .017 and $.016 \mathrm{sec} /$ bit; intercepts were .487 and $.489 \mathrm{sec}$. For purposes of statistical analysis, the 12 pigeons were compared with the 11 human subjects. Intercepts for the pigeons were significantly higher than those for the humans $[F(1,21)=$ $32.946, p=.0000$ ], and slopes for the pigeons were significantly lower $[F(1,21)=4.418, p=.0478]$.

\section{EXPERIMENT 5 Human "Peck" Reaction Times}

Evidence in the human literature indicates that the type of stimulus and of response influences slope. Teichner and Krebs (1974) showed that when the stimulus was audibly naming the number of a response button, both intercepts and slopes were higher than those in the more common case in which the stimulus was a light just above the button (see also ten Hoopen, Akerboom, \& Rayymakers, 1982). On the other hand, when the response was to name the presented stimulus, the slopes were lower than when an associated button had to be pushed (Theios, 1975). In Experiments 1-4, the stimuli presented to people and to pigeons were identical, but the responses differed: $\mathrm{Pi}-$ geons pecked, whereas people touched. Associated with this difference was the fact that the pigeon's head was much closer to the monitor than was the human subject's. It is, of course, difficult, if not impossible, to equate the responses in terms of topography, difficulty, naturalness, and other dimensions. We chose, therefore, a response for the human subjects that was topographically similar to the pigeon's-namely a "peck" at the screen. The main question is whether the human slopes would differ as a function of response topography.

\section{Method \\ Subjects. Ten subjects, of the same age range as those in Exper- iment 4, were used. Three of the subjects had participated in Exper- iment 4,5 volunteered to participate, and the other 5 were each paid $\$ 5$. \\ Apparatus. The apparatus was identical to that in Experiment 4, with one exception. A plastic animal "beak" (purchased in a toy store) was attached to the subject's face atop the nose and was held in place by an elastic strap around the head. The beak was $11.2 \mathrm{~cm}$ long, from open base to tip. \\ Procedure. The procedure was identical to that in Experiment 4 , with two exceptions. First, "pecks" of the beak were required, rather than touches of the index finger. Second, because pecking was an unusual response for the human subjects, the preliminary training phase, in which the subjects responded to random black squares, was permitted to last as long as the subject chose. The subjects spent less than $2 \mathrm{~min}$ in this preliminary phase.}

\section{Results and Discussion}

The results are shown by the downward pointing triangles in Figure 3. The linear fit accounted for $90 \%$ of the variance, and thus, Hick's law holds, whether people touch squares or peck them. The individual $r$ values were 77 , $.85, .88, .89, .94, .95, .96, .97, .97$, and 1.00 . Compared with the touch responses in Experiment 4, the peck responses in the present experiment led to higher slopes $(.044 \mathrm{sec} / \mathrm{bit}$ in the present experiment and .034 in Experiment 4), although the difference was not statistically significant. Intercepts increased from .293 in Experiment 4 to .407 in the present experiment, this difference being statistically significant $[F(1,19)=4.790, p=.0413]$.

We compared pigeons with people when both pecked the monitor. The difference in intercepts between the two species was no longer statistically significant $[F(1,20)=$ $2.685, p=.1169]$. The pigeon slopes were again significantly lower than the human peck slopes $[F(1,20)=12.016$, $p=.0024]$.

\section{GENERAL DISCUSSION}

We asked three questions, the first of which was whether Hick's law describes choice RTs in pigeons. The answer is yes. Experiments 1-3 demonstrated linear relationships between RT and $\log _{2}$ number of potential targets, or bits of information. Thus, the same relationship is observed in people, monkeys, and pigeons.

The second question was whether parameters of the Hick function were affected by training. Jensen (1982) concluded that "We have found no indication of practice effects in any of the samples tested ... either across trials in a single session or across sessions from day to day" (p. 280). Eysenck (1987) concurred, writing that "There is an absence of practice effect, which supports the hypothesis that the RT measure does not involve anything that could be called learning, association, ... memory scanning ... [or] ... cognitive strategies in the usual sense of the term" (p. 290). However, in an earlier review of the literature on visual choice RT, Teichner and Krebs (1974) showed that practice caused both intercepts and slopes to decrease (see also Longstreth, 1984; Luce, 1986; Widaman \& Carlson, 1989). Jensen (1987) came to conclude that training did not matter in his experiments, where relatively few trials were provided. But in order to interpret RT-IQ correlations, it is important to know whether training of any length affects the function parameters of Hick's law. In the present research with pigeons, intercepts and slopes decreased with training, this being especially clear when percentile reinforcement contingencies selectively reinforced fast responding. The decrease in intercept occurred consistently across the three experiments with pigeons, whereas the decrease in slope was noisier and, in some cases, did not attain statistical significance. One possible reason was that the slopes were low to begin with, and thus, a floor effect might have been involved. In general, the results for pigeons are consistent with Teichner and Krebs (1974).

The third question was whether slopes for pigeons were higher than those for humans. This question was motivated by some researchers' use of choice RT to support inherited differences in intelligence across human individuals and races. We reasoned that, if parameters of the Hick function in fact represent heritable differences in intelligence, these should be manifest across species. Because pigeons are 
generally thought to be less intelligent than people, we predicted that slopes for pigeons would be higher than those for humans. No predictions were made with respect to intercept, because intercepts are influenced by the particulars of procedure, such as travel distance between the center response and the PT, and by the physical attributes of the subjects.

Slopes for pigeons were found to be significantly lower than those for humans when people touched the targets. Because the literature indicates that different types of responses may result in different slopes (e.g., Theios, 1975), we also studied RTs when people "pecked" the targets and found that the slopes for pigeons were again significantly lower than those for humans (Experiment 5). Thus, if slopes are inversely related to intelligence and this finding has cross-species generality, then pigeons are more intelligent than people. We consider alternative interpretations below.

In Jensen's studies, human subjects were required to press a center button until a PT became a target, and, therefore, he was able to distinguish between the time taken to lift the finger from the center button and the time taken to move the finger from the center button to the target. In our case, because the pigeon response is ballistic, we could not separate the two. Since movement-time functions are relatively flat in humans and tend to show the same relationship with IQ as lift time, the present comparisons with pigeons are possible.

Jensen (1980) proposed that another measure-namely, within-subjects variability - was also inversely related to intelligence (see also Jensen, 1987). He calculated standard deviations $(S D s)$ at each PT for each subject and then calculated a group mean for each PT. Since $S D$ s are affected by the magnitude of the mean, Jensen's $S D$ s did not provide an independent statistic. In any case, he concluded that the greater the $S D$, the lower the intelligence. Were pigeons more variable (higher $S D$ s) than people under the Hick task? To control for differences in magnitudes of the means, we calculated within-subjects coefficients of variation. We considered the first 40 responses for all the pigeons in Experiments 1 and 3 and compared them with the human subjects in Experiment 4 (touch PT). Because outlying values greatly influence both $S D$ and coefficient of variation and because these were the first 40 responses and, therefore, included a number of long pauses in many cases, we excluded the longest five RTs from our calculations. Thus, we estimated coefficients of variations based on the fastest 35 of 40 responses per PT for pigeons and people. The group $\times$ bit interaction was significant $[F(3,63)=$ $10.951, p=.0000]$, with human subjects showing significantly lower coefficients of variation at the 1-, 2-, and 3-bit values. We also compared the human data with the pigeon's terminal 40 responses. Again, the group $\times$ bit interaction was highly significant $[F(3,63)=22.722, p=$ $.0000]$, but now the only difference was at the 0 -bit condition, where human coefficients of variation were significantly higher than those for the pigeons. Similar results were obtained when the human peck data were compared with the data for the pigeons. Thus, with respect to coefficients of variation, a conservative conclusion is that it is not clear that pigeons are more variable than people.

One additional contribution of the present research was to demonstrate the usefulness of a procedure that selectively reinforced rapid-as-possible RTs - namely, percentile reinforcement (Galbicka, 1994). Under these contingencies in Experiment 3, the subjects were reinforced for only the shortest $70 \%$ of their RTs over the recent past, thereby continually pressuring them to respond as fast as possible. The slopes and intercepts of the Hick function decreased across training. In measures of intelligence more generally, explicit contingencies may be useful. When contingencies are vague or unspecified, differences in performance may reflect differences in perceived demands and consequences.

The present findings extend the generality of Hick's law as a description of choice RT to pigeons. As with human subjects, orderly data are obtained from pigeons. However, the results also raise questions about whether choice $\mathrm{RT}$ is a useful measure of intelligence. Most important are three findings: (1) the slopes and intercepts of the RT versus PT functions decreased with training; (2) the type of response affected the slope and the intercept; and (3) the slopes for the pigeons were lower than those for the humans. The first two findings are important in evaluating the claim that intelligence has a major unmodifiable component. Choice RT was thought to be a good measure of intelligence, because both RT and IQ did not depend on experience (Eysenck, 1987; Jensen, 1980). Consistent with other findings in the literature (Longstreth, 1986; Luce, 1986; Teichner \& Krebs, 1974), the present results show that RT functions are influenced by training and response topography.

Explanations of the third result - slopes for pigeons are lower than those for humans - could take one of four forms: (1) the procedures were insensitive, and therefore, the data are misleading; (2) pigeons use a different tactic (or method of responding) than do human subjects; (3) pigeons are more intelligent than people; or (4) slopes of the Hick function do not provide a useful measure of heritable general factor intelligence. We discuss each of these.

1. The orderly functions obtained from pigeons and people support the adequacy of the procedures, as do the significant influences of parametric manipulations. The precision of the recording apparatus might have influenced the slopes, but the apparatus was identical for both pigeons and people. Thus, it is unlikely that differences between the slopes for pigeons and humans were caused by inadequacies in the procedure.

2. We are aware of no evidence that different response tactics underlie complex choice RTs in pigeons and people. There is evidence, to the contrary, that pigeons use modes of serial processing that are similar to those of humans (e.g., Roberts, 1987). Furthermore, if Hick functions differ between pigeons and people because of different ways of confronting the task, the same might be the case for individuals, groups, or races. Response tactics, 
not intelligence, would then account for RT effects (see Detterman, 1987).

3 . In an influential paper on the comparative psychology of intelligence, Macphail (1987) writes, "It was-as it still is - generally accepted that man is the most intelligent of organisms" (p. 645). If intelligence is taken to be a function of a general heritable $g$ factor-and Hick's parameters are valid measures - then the counterintuitive conclusion follows that pigeons are more intelligent than people. An alternative view assumes that different intelligences or factors are employed in different situations by different individuals, groups, and species (see Carlson \& Widaman, 1987; Gardner, 1983; Sternberg, 1985). The theory of multiple intelligences is compatible with the hypothesis that, because of their evolutionary histories, pigeons respond faster than people in some complex choice situations. To survive, pigeons forage, often in flocks, for small pieces of grain, as well as for insects that move and fly. Such foraging requires rapid choices to be made from simultaneously available objects. Thus, the pigeon might, in fact, be capable of more rapid choices among potential food items than are people. According to this interpretation, Hick functions show that pigeons are more adept at choosing among concurrently available targets (at least in the type of situation studied) but indicate nothing about general capabilities or $g$.

(4) Taken together, our results do not support complex RT as an index of heritable general intelligence. Parameters are affected by training, thus arguing against heritability; and functions for pigeons indicate an intelligence that is higher than that of humans, thus arguing against a generally valid measure.

\section{REFERENCES}

BeAuduCEL, A., \& BROCKE, B. (1993). Intelligence and speed of information processing: Further results and questions on Hick's paradigm and beyond. Personality \& Individual Differences, 15, 627-636.

Carlson, J. S., \& Widaman, K. F. (1987). Elementary cognitive correlates of $G$ : Progress and prospects. In P. A. Vernon (Ed.), Speed of information-processing and intelligence (pp. 69-99). Norwood, NJ: Ablex.

CARroll, J. B. (1987). Jensen's mental chronometry: Some comments and questions. In S. Modgil \& C. Modgil (Eds.), Arthur Jensen: Consensus and controversy (pp. 297-307). New York: Falmer Press.

DetTERMAN, D. K. (1987). What does reaction time tell us about intelligence? In P. A. Vernon (Ed.), Speed of information-processing and intelligence (pp. 177-200). Norwood, NJ: Ablex.

EYSENCK, H. (1987). Intelligence and reaction time: The contribution of Arthur Jensen. In S. Modgil \& C. Modgil (Eds.), Arthur Jensen: Consensus and controversy (pp. 285-295), New York: Falmer Press.

GALBICKA, G. (1994). Shaping the 21 st century: Moving percentile schedules into applied settings. Journal of Applied Behavior Analysis, 27, 739-760.

GARDNER, H. (1983). Frames of mind: The theory of multiple intelligences. New York: Basic Books.

HICK, W. E. (1952). On the rate gain of information. Quarterly Journal of Experimental Psychology, 4, 11-26.

HYMAN, R. (1953). Stimulus information as a determinant of reaction time. Journal of Experimental Psychology, 45, 188-196.

Jensen, A. R. (1980). Bias in mental testing. New York: Free Press.

JENSEN, A. R. (1982). The chronometry of intelligence. In R. J. Sternberg (Ed.), Advances in the psychology of human intelligence (pp. 255310). Hillsdale, NJ: Erlbaum.

JENSEN, A. R. (1987). Individual differences in the Hick paradigm. In P. A. Vernon (Ed.), Speed of information-processing and intelligence (pp. 101-175). Norwood, NJ: Ablex.

Jensen, A. R., \& MUNRo, E. (1979). Reaction time, movement time, and intelligence. Intelligence, 3, 121-126.

KORNBLUM, S. (1969). Sequential determinants of information processing in serial and discrete choice reaction time. Psychological Review, 76, 113-131.

LAURSEN, A. M. (1977). Task dependence of slowing after pyramidal lesions in monkeys. Journal of Comparative \& Physiological Psychology, 91, 897-906.

LONGSTRETH, L. E. (1984). Jensen's reaction-time investigations of intelligence: A critique. Intelligence, 8, 139-160.

LUCE, R. D. (1986). Response times: Their role in inferring elementary mental organization. New York: Oxford University Press.

MACPHAIL, E. M. (1987). The comparative psychology of intelligence. Behavioral \& Brain Sciences, 10, 645-696.

Neubauer, A. C. (1990). Speed of information processing in the Hick paradigm and response latencies in a psychometric intelligence test. Personality \& Individual Differences, 11, 147-152.

Platt, J. R. (1973). Percentile reinforcement: Paradigms for experimental analysis of response shaping. In G. H. Bower (Ed.), The psychology of learning and motivation: Advances in theory and research (Vol. 7, pp. 271-296). New York: Academic Press.

POSNER, M. I. (1986). Chronometric explorations of mind. New York: Oxford University Press.

ROBERTS, S. (1987). Evidence for distinct serial processes in animals: The multiplicative-factors method. Animal Learning \& Behavior, 15, 135-173.

STERnBerg, R. J. (1985). Beyond IQ. Cambridge: Cambridge University Press.

TEICHNER, W. H., \& KREBS, M. J. (1974). Laws of visual choice reaction time. Psychological Review, 81, 75-98.

ten Hoopen, G., Akerboom, S., \& RaAymakers, E. (1982). Vibrotactual choice reaction time, tactile receptor systems and ideomotor compatibility. Acta Psychologica, 50, 143-157.

THEIOS, J. (1975). The components of response latency in simple human information processing tasks. In P. M. A. Rabbitt \& S. Dornic (Eds.), Attention and performance $V$ (pp. 418-440). New York: Academic Press.

Widaman, K. F., \& Carlson, J. S. (1989). Procedural effects on performance on the Hick paradigm: Bias in reaction time and movement time parameters. Intelligence, 13, 63-85.

(Manuscript received December 8, 1998; revision accepted for publication June 28,1999 .) 